Proceedings of the Online Conference "Applications of Physics in Mechanical and Material Engineering"

\title{
What is the Physical Meaning of Thermodynamic Critical Field?
}

\author{
K.P. Kosk-JonieC ${ }^{a, *}$, M. KostrzewA ${ }^{a}$, \\ K. $\mathrm{KROK}^{a}$ AND R. SzCZÉŚNIAK ${ }^{a, b}$ \\ ${ }^{a}$ Jan Dlugosz University in Częstochowa, 42-201 Częstochowa, Poland \\ ${ }^{b}$ Częstochowa University of Technology, 42-201 Częstochowa, Poland
}

Doi: 10.12693/APhysPolA.139.555

*e-mail: kamilakosk@gmail.com

\begin{abstract}
Based on our own research on thermodynamic properties of superconductors and a detailed review of the available scientific publications on this topic, we have created a detailed analysis of the thermodynamic critical field. The following publication allows to obtain a broader view of the importance of the thermodynamic critical field in the process of searching for new superconducting materials with the desired physical parameters. Understanding the thermodynamic critical field parameter is key to comparing the thermodynamic properties of superconductors and could lead to a better understanding of the phenomenon of superconductivity. It is especially important in terms of comparing the experimental data with the data obtained using theoretical methods.
\end{abstract}

topics: superconductivity, thermodynamic properties, thermodynamic critical field

\section{Introduction}

In the 1950s, starting from the general theory of phase transitions of the second kind, Ginzburg and Landau presented a theory describing the properties of superconductors. To this day, the equations derived by these two Russian scientists are used by everyone who deals with the theory of superconductivity. This developed theory allowed for a very broad description of the thermodynamic properties of superconductors, with particular emphasis on the second type of superconductors. The Ginzburg and Landau theory laid the foundations for the microscopic theory of BCS superconductivity.

Many years of research by Ginzburg and Landau allowed them finding, for example, the possibility of assigning the phase transitions to order parameter and the conversion of the thermodynamic potential into a power series by its use. Moreover, Ginzburg and Landau noticed that at a certain critical value of the magnetic field $H_{c}$, a superconducting-normal transition can be observed.

In 1957, Bardeen, Cooper and Schriffer began a new chapter in conductivity research, namely the microscopic theory of superconductivity (BCS). This theory was used and significantly extended by Eliashberg, thanks to which in its present form it allows to determine the thermodynamic properties of superconductors in which there are strong interactions of electrons with the phonon lattice [1].

Many modern scientists have attempted to work with Eliashberg's equations to study new superconductors [2]. The presented results obtained on the basis of these equations are much closer to the conducted experiments than the previous applications of the BCS theory itself. When taking into account the strong electron-phonon pairing and the vertex corrections, it is possible to obtain results with very high accuracy.

\section{Thermodynamic critical field}

From the many described parameters, this time we focused on the thermodynamic critical field. Applying the Ginzburg-Landau theory, it is possible to estimate the upper critical field for a superconductor. We should then look at this parameter more broadly. In the case of the analysis of the first type of superconductors, we observe a transition between the normal and superconducting state, induced by the magnetic field $H_{c}$, and this is the first type of transition. According to current knowledge, there are two types of the thermodynamic critical field.

The thermodynamic critical field is expressed by the relation

$$
H_{c}(T)=\frac{a}{\sqrt{2 b \mu_{0}}}\left(T_{c}-T\right),
$$

while the second critical field of type II superconductors is described by

$$
H_{c 2}(T)=\sqrt{2} \kappa H_{c}(T)=\frac{1}{2 \pi \mu_{0} \xi^{2}} \phi_{0},
$$

with

$$
\kappa=\frac{\lambda_{L}}{\xi}=\frac{m_{e}}{e \hbar} \sqrt{\frac{4 b}{\mu_{0}}},
$$




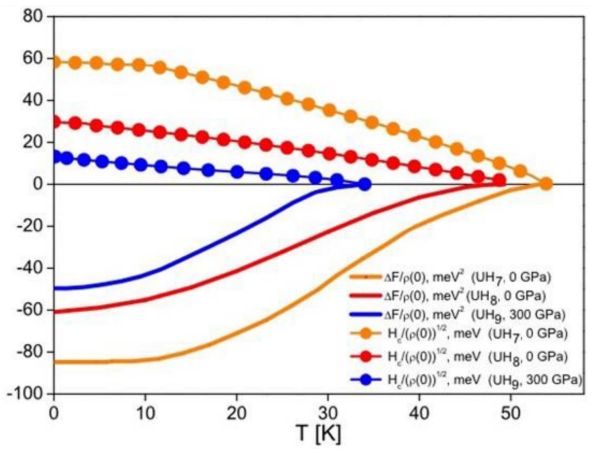

Fig. 1. Thermodynamic critical fields and free energy difference between the normal and superconducting states for $\mathrm{UH}_{x}$ systems.

where $\lambda_{L}$ - the dependence of the depth of penetration of magnetic field induction on temperature, $\kappa$ - the Ginzburg-Landau parameter which helps to determine the type of superconductor, and $\mu_{0}$ is the magnetic permeability of vacuum [3]. A very important observation in our research is the fact that the temperature dependence of the critical field near $T_{c}$ is a linear function and at the phase transition temperature it reaches exactly zero $[4,5]$.

In our studies using the Eliashberg formalism, the thermodynamic critical field $H_{c}$ is calculated using

$$
\frac{H_{c}}{\sqrt{\rho(0)}}=\sqrt{-8 \pi\left(\frac{\Delta F}{\rho(0)}\right)},
$$

where $\rho(0)$ is the electron density of states in the Fermi energy.

In our research, we also calculated the difference between the free energy $\Delta F$ in the normal and superconducting states. This is expressed as

$$
\begin{aligned}
& \frac{\Delta F}{\rho(0)}=-\frac{2 \pi}{\beta} \sum_{n=1}^{M}\left(\sqrt{\omega_{n}^{2}+\Delta_{n}^{2}}-\left|\omega_{n}\right|\right) \\
& \quad \times\left(Z_{n}^{S}-\frac{\left|\omega_{n}\right|}{\sqrt{\omega_{n}^{2}+\Delta_{n}^{2}}} Z_{n}^{N}\right) .
\end{aligned}
$$

Here, $Z_{n}^{S}$ and $Z_{n}^{N}$ are the wave normalization function factors.

We performed our calculations for three uranium-hydrogen compounds based on research of Kruglov et al. [6]. The calculation results of the free energy and thermodynamic critical field differences are presented in Fig. 1.

As previous studies show [7-9], hydrogenated compounds can be expected to have a high critical temperature to become superconducting at a relatively low operating pressure. As we have also shown in our earlier works, the formalism of the Eliashberg equations applied to compounds with a strong electron-phonon correlation gives excellent results, unlike other calculation formulas which often lead to an underestimation of the critical temperature.

One can see clearly in Fig. 1 that both the free energy difference and the thermodynamic critical field reach zero at the critical temperature. The free energy dependencies are marked with solid lines: the orange line for $\mathrm{UH}_{7}$, the red one - for $\mathrm{UH}_{8}$, and the blue line for $\mathrm{UH}_{9}$. Analogously, the corresponding values of the thermodynamic critical field were marked by means of orange, red and blue circles.

\section{Conclusions}

The Eliashberg equations play a key role in the theoretical considerations about the superconducting state at the present moment. Their modifications, such as vertex corrections, allow for obtaining better and better theoretical models and thus are helpful in the search for high-temperature superconductors.

The thermodynamic parameters obtained in this way are the closest to the experimental data as opposed to other methods. Conducting research taking into account the thermodynamic parameter of the critical field is necessary to check the induction of the superconducting state. It is also worth paying attention to the behavior of superconductors in a magnetic field. Namely, when a strong magnetic field with a value above $\mathrm{H}_{c 2}$ is applied to a type II superconductor, its transition to a normal state can be observed. If then the value of the magnetic field applied to this superconductor was gradually reduced, a transition into a mixed state could be observed and this transition would be a type II transition.

In the studies on uranium hydrides described above, as well as in the previous studies focused on lead, the course of the thermodynamic plot of the critical field with the same characteristic curve shape can be observed.

\section{References}

[1] J. Bardeen, L.N. Cooper, J.R. Schrieffer, Phys. Rev. 108, 1175 (1957).

[2] G.M. Eliashberg, Sov. Phys. JETP 11, 696 (1960).

[3] M.W. Jarosik, R. Szczęśniak, D. Szczęśniak, Acta Phys. Pol. A 118, 1031 (2010).

[4] J.P. Carbotte, Rev. Mod. Phys. 62, 1027 (1990).

[5] J.W. Ekin, Supercond. Sci. Technol. 23, 083001 (2010).

[6] I.A. Kruglov, D.V. Semenok, H. Song, Phys. Rev. B 4, 10 (2018).

[7] K.P. Kosk-Joniec, I.A. Wrona, M. Kostrzewa, R. Szczęśniak, Acta Phys. Pol. A 138, 181 (2020).

[8] M.W Jarosik, I.A. Wrona, A.M. Duda, Solid State Commun. 219, 1 (2015).

[9] D. Szczęśniak, I.A. Wrona, E.A. Drzazga, A.Z. Kaczmarek, K.A. Szewczyk, J. Phys. Condens. Matter 29, 445602 (2017). 